\title{
Effect of Solid-State Extrusion on Melting Behavior and Morphology of High-Density Polyethylene
}

\author{
K. Dhawan, D. R. Chaubey, Y.S. Yadav, \\ P. C. JAIN, and V. S. NANDA \\ Department of Physics and Astrophysics, \\ University of Delhi, Delhi-110007, India.
}

(Received October 22, 1979)

\begin{abstract}
The effect of solid-state extrusion on the melting behavior of high-density polyethylene has been investigated in detail. It was found that $\Delta T_{\mathrm{m}}$, the increase in melting temperature, and $\Delta H_{\mathrm{f}}$, the increase in the heat of fusion, both tend to saturate for values of the extrusion ratio $R>20$. We have also determined the orientation in the crystalline and the amorphous phases through $\mathrm{X}$-ray and birefringence measurements. It was found that the orientation function $f_{\mathrm{am}}$ for the amorphous phase also saturates for $R>20$. A careful analysis of our results has brought out that, irrespective of the temperature at which the extrudate is prepared, a universal relationship exists between $\Delta T_{\mathrm{m}}, \Delta H_{\mathrm{f}}$, and $f_{\mathrm{am}}$. It was further found that when an oriented sample is completely etched, it undergoes fusion at the normal melting temperature of the unoriented polymer. All these findings point to the conclusion that the changes in the melting behavior of high-density polyethylene are brought about essentially by the orientation produced in the amorphous phase.

KEY WORDS Polyethylene / Solid-State Extrusion / Melting Characteristics / DSC / Birefringence / X-Rays / Orientation / Crystalline Phase / Amorphous Phase / Etching /
\end{abstract}

In a semicrystalline polymer, melting is a complex process affected by molecular weight distribution, ${ }^{1}$ crystallinity, ${ }^{2}$ and the morphology ${ }^{3}$ of the sample. Thus, any physical process which brings about structural changes in a polymer inevitably affects its melting characteristics. The effect of drawing on the melting temperature and the heat of fusion of polyethykene has been investigated by Sumita et al., ${ }^{4}$ who showed that changes in the melting characteristics are caused mainly by orientation produced in the amorphous phase. Recently, Kojima et $a l^{5}$ reported similar results in the solid-state extrusion of high-density polyethylene. However, in contrast to Sumita et al., ${ }^{4}$ they attribute the increase in melting temperature and heat of fusion to an increase in crystallinity. In order to study the problem in greater detail, we have carried out the solid-state extrusion of high-density polyethylene. Not only the melting characteristics of the extrudates but also the orientation effects in their crystalline and amorphous phases have been investigated through $\mathrm{X}$-ray and birefringence measurements. It was observed that for a deformation ratio of about 20 , the melting temperature, the heat of fusion, and the orientation of amorphous chains along the flow direction tend to saturate. On interpreting the results, we tend to agree with Sumita et al., ${ }^{4}$ that the changes in the melting behavior are caused mainly by orientation produced in the amorphous phase.

\section{EXPERIMENTAL}

\section{Sample Preparation}

The high-density polyethylene used in the present experiment was of commercial grade, GD 6260, supplied by Hoechst Chemicals Ltd., India. The quoted density of the granules of this material is $0.960 \mathrm{~g} \mathrm{~cm}^{-3}$ and the melt index is $0.3 \mathrm{~g} \mathrm{~min}^{-1}$. On measuring the viscosity-average molecular weight, the value was found to be $1.24 \times 10^{5}$. The actual samples employed in the solid-state extrusion were prepared by recrystallizing the granules in a highpressure cylinder fitted with a conical die. The 
granules were first brought to the molten state and then cooled to $398 \mathrm{~K}$. To accelerate the process of crystallization, a pressure undercooling of $2 \mathrm{kbar}$ was employed. After an hour, the sample was cooled to the desired extrusion temperature. The density of this sample at the room temperature was found to be $0.9710 \mathrm{~g} \mathrm{~cm}^{-3}$ which corresponds to the 82 percent weight fraction crystallinity, taking the crystal and amorphous specific volumes ${ }^{6,7}$ respectively to be equal to 0.9995 and $1.1755 \mathrm{~cm}^{3} \mathrm{~g}^{-1}$. The details regarding the extrusion procedure are given elsewhere. ${ }^{8}$ The effects of various parameters, such as the die angle, capillary bore, capillary length, and extrusion temperature etc. were investigated. The best extrudates were obtained by using a conical die having a $42^{\circ}$ cone angle, a 3 to $5 \mathrm{~mm}$ capillary bore and an approximately $40 \mathrm{~mm}$ length. In every case, the extrudate obtained was in the form of a continuous rod. Due to the conical geometry of the die, the extrusion or deformation ratio increased continuously along the length of the extrudate and acquired a constant value after the material, initially in the conical portion of the die, had been completely extruded. Oriented samples of different extrusion ratios were obtained by cutting thin slices normal to the flow direction at various points from the initial part of the extrudate.

\section{DSC Measurements}

A Perkin-Elmer Differential Scanning Calorimeter (DSC-1B) was used with varying sample sizes ( 3 to $10 \mathrm{mg}$ ) and different heating rates ( 2 to $16 \mathrm{~K} \mathrm{~min}^{-1}$ ). The melting temperature $T_{\mathrm{m}}$ and the heat of fusion, $H_{\mathrm{f}}$ were determined, respectively from the location of the peak maximum and the area under the peak of the DSC thermograph. Indium $\left(T_{\mathrm{m}}=419 \mathrm{~K}\right.$ and $\left.H_{\mathrm{f}}=6.8 \mathrm{calg}^{-1}\right)$ was employed as the standard for calibration.

\section{Determination of Crystalline and Amorphous Orientation}

Birefringence $\Delta n$ in samples of various extrusion ratios was measured by means of the retardation method, using a polarizing microscope. The results are shown in Table I. For determining the degree of orientation along the crystal $c$-axis, wide-angle Xray diffraction patterns were taken, using a flat plate camera. The orientation function, $f_{\mathrm{c}}$, was then evaluated by using Herman's type orientation function. For this purpose, azimuthal scannings of
(200) and (020) reflections were carried out and from which $\left\langle\cos ^{2} \Phi_{200}\right\rangle$ and $\left\langle\cos ^{2} \Phi_{020}\right\rangle$ were evaluated by the usual procedure. ${ }^{9}$ The degrees of $a$-, $b$-, and $c$-axis orientations, denoted respectively by $f_{a}, f_{b}$, and $f_{c}$ were then computed from the relations,

$$
\begin{aligned}
& f_{a}=\frac{1}{2}\left(3\left\langle\cos ^{2} \Phi_{200}\right\rangle-1\right) \\
& f_{b}=\frac{1}{2}\left(3\left\langle\cos ^{2} \Phi_{020}\right\rangle-1\right) \\
& f_{c}=-\left(f_{a}+f_{b}\right)
\end{aligned}
$$

The crystalline birefringence $\Delta n_{\mathrm{c}}$ was determined using the result,

$$
\Delta n_{\mathrm{c}}=f_{\mathrm{c}} \Delta n_{\mathrm{c}}^{0}
$$

where $\Delta n_{\mathrm{c}}^{0}$ is the intrinsic birefringence value of the ideally oriented crystal. Knowing the total birefringence and the birefringence, $\Delta n_{c}$, of the crystalline phase, the firefringence, $\Delta n_{a}$, of the amorphous phase was obtained from the relation, ${ }^{10}$

$$
\Delta n_{a}=\left(\Delta n-X_{\mathrm{v}} \Delta n_{\mathrm{c}}\right) /\left(1-X_{\mathrm{v}}\right)
$$

where $X_{\mathrm{v}}$ is the degree of volume-fraction crystallinity connected with the weight-fraction crystallinity of the sample by the relation,

$$
X_{v}=\frac{\rho}{\rho_{\mathrm{c}}} X
$$

where $\rho$ and $\rho_{\mathrm{c}}$ denote the sample and the crystal phase densities respectively. The values of $\Delta n_{\mathrm{c}}$ and $\Delta n_{a}$ thus obtained are also given in Table I. The amorphous orientation function, $f_{\text {am }}$ was determined from the relation, ${ }^{12}$

$$
f_{\mathrm{am}}=\frac{\Delta n_{\mathrm{a}}}{\Delta n_{\mathrm{a}}^{0}}
$$

In the foregoing calculations, we took $\Delta n_{\mathrm{c}}^{0}=0.0584$ while the value of $\Delta n_{\mathrm{a}}^{0}$ was determined from the relation, ${ }^{12}$

$$
\Delta n_{\mathrm{a}}^{0}=\frac{\rho_{\mathrm{a}}}{\rho_{\mathrm{c}}} \Delta n_{\mathrm{c}}^{0}
$$

where $\rho_{\mathrm{a}}$ denotes the density of the amorphous phase. The contribution from the "form birefringence" was neglected. ${ }^{13}$

\section{Nitric Acid Etching}

Etching of the oriented samples was carried out 
Table I. Variation in the birefringence of the material $\Delta n$, birefringence of the crystalline phase $\Delta n_{\mathrm{c}}$, and birefringence of the amorphous phase $\Delta n_{\mathrm{a}}$ with extrusion ratio $R$ (columns (a) and (b) give values for extrudates obtained at $353 \mathrm{~K}$ and $388 \mathrm{~K}$, respectively)

\begin{tabular}{|c|c|c|c|c|c|c|}
\hline \multirow{2}{*}{$R$} & \multicolumn{2}{|c|}{$\Delta n \times 10^{3}$} & \multicolumn{2}{|c|}{$\Delta n_{\mathrm{c}} \times 10^{3}$} & \multicolumn{2}{|c|}{$\Delta n_{\mathrm{a}} \times 10^{3}$} \\
\hline & (a) & (b) & (a) & (b) & (a) & (b) \\
\hline 1 & 0.0 & 0.0 & 0.0 & 0.0 & 0.0 & 0.0 \\
\hline 2.5 & 22.6 & 22.6 & 27.5 & 28.0 & 3.5 & 2.3 \\
\hline 5 & 36.8 & 36.8 & 43.0 & 44.3 & 13.6 & 8.6 \\
\hline 7.5 & 43.0 & 43.0 & 49.5 & 50.9 & 18.5 & 13.3 \\
\hline 10 & 46.8 & 46.8 & 52.6 & 54.4 & 25.0 & 17.9 \\
\hline 15 & 48.8 & 48.8 & 53.1 & 54.8 & 33.5 & 26.0 \\
\hline 20 & - & 51.3 & - & 54.8 & - & 38.0 \\
\hline 25 & - & 52.2 & - & 54.9 & - & 41.6 \\
\hline 30 & - & 52.2 & - & 54.9 & - & 42.0 \\
\hline
\end{tabular}

with 100 percent fuming nitric acid. Sample chips 2 to $3 \mathrm{~mm}$ thick were sealed with an excess of nitric acid in a specially designed glasstube and kept at $335 \mathrm{~K}$ for various periods of time. After etching, the samples were washed with distilled water and then by acetone for 8 hours. The samples were then kept at $330 \mathrm{~K}$ for about 12 hours to remove all traces of moisture.

\section{RESULTS}

In the present study, both the melting temperature $T_{\mathrm{m}}$ and the heat of fusion $H_{\mathrm{f}}$ were found to increase with $R$, attaining an almost constant value for sufficiently large extrusion ratios. It was found that the effect of the sample size, within the range specified earlier ( 3 to $10 \mathrm{mg}$ ), on $T_{\mathrm{m}}$ and $H_{\mathrm{f}}$ was negligible. However these parameters were found to vary with the heating rate (H.R.). In Figure 1, the variations of $T_{\mathrm{m}}$ with H.R. are shown for two samples ( $R=1$ and $R=25$ ). It may be noted that the extent of superheating in both cases is the same. Thus $\Delta T_{\mathrm{m}}$, which represents the increase in melting temperature due to extrusion with respect to the untreated sample, is independent of the heating rate. A similar result was found to apply in the case of $\Delta H_{\mathrm{f}}$, which represents the increase in heat of fusion due to extrusion. Hence, the extent of orientation brought about by extrusion may best be represented in terms of $\Delta T_{\mathrm{m}}$ and $\Delta H_{\mathrm{f}}$.

In Figure 2, the variations in $\Delta T_{\mathrm{m}}$ and $\Delta H_{\mathrm{f}}$ with $R$ are shown for extrudates obtained at $388 \mathrm{~K}$. It is

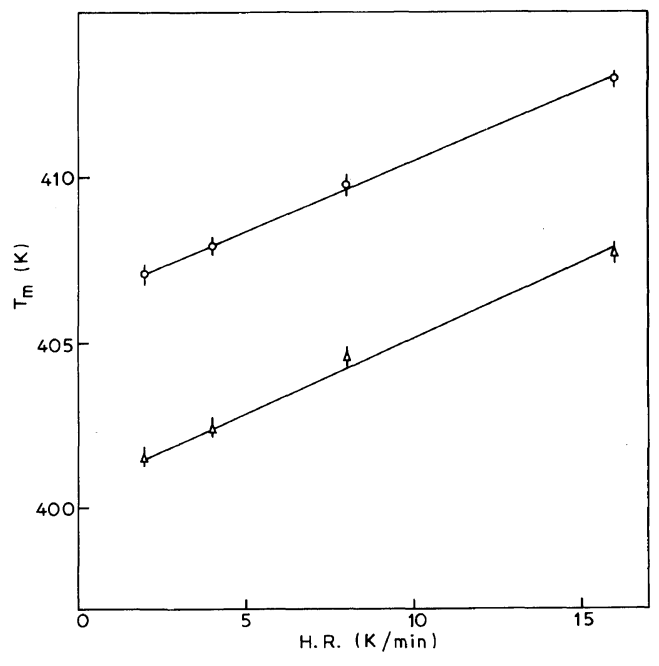

Figure 1. Variation of $T_{\mathrm{m}}$ with heating rate H.R.: lower curve, unextruded material $(R=1)$; upper curve, $R=25$.

noted that in both cases the increase is linear for small $R$, while for $R>20$, the increase is almost negligible. Further, for a given $R, T_{\mathrm{m}}$ was found to depend on extrusion temperature $T_{\text {exi }}$. Such a dependence on $R=15$ is shown in Figure 3. It is seen that with an increase in $T_{\text {ext }}, T_{\mathrm{m}}$ increases at first and, after showing a flat maximum, decreases for temperatures close to $T_{\mathrm{m}}$. A similar trend is observed for the dependence of $H_{\mathrm{f}}$ on $T_{\mathrm{ext}}$ and is shown in Figure 4 for $R=15$. From the similarity of 


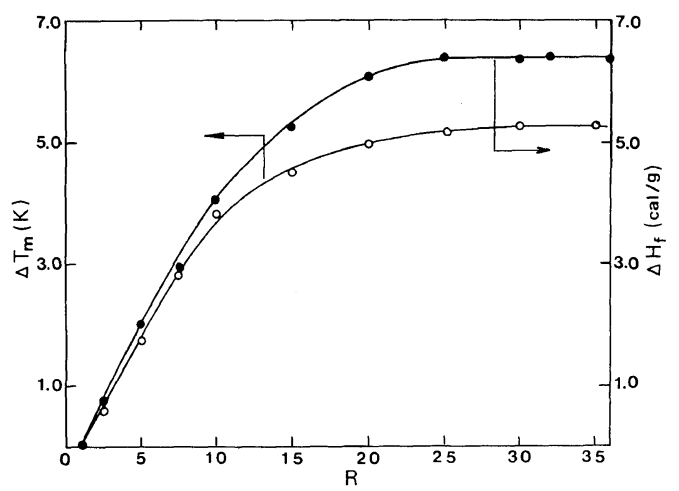

Figure 2. $\Delta T_{\mathrm{m}}$ and $\Delta H_{\mathrm{f}}$ as function of extrusion ratio $R: \bigcirc, \Delta T_{\mathrm{m}}$ values; $\bigcirc, \Delta H_{\mathrm{f}}$ values.

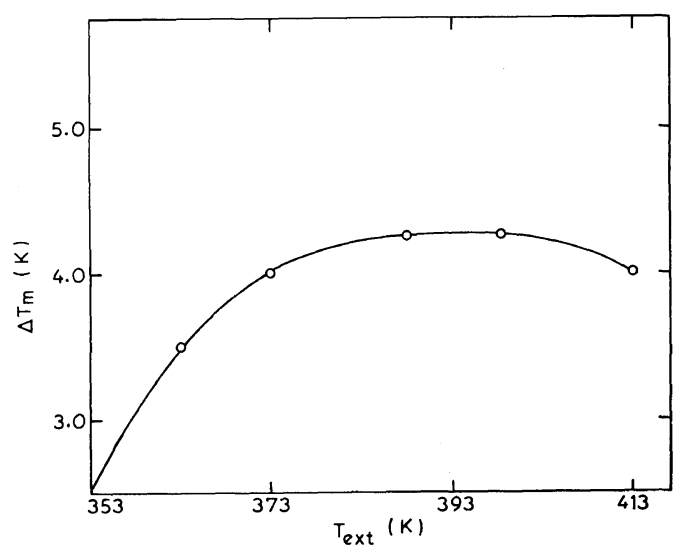

Figure 3. $\Delta T_{\mathrm{m}}$ as a function of extrusion temperature $T_{\mathrm{ext}}$ for $R=15$.

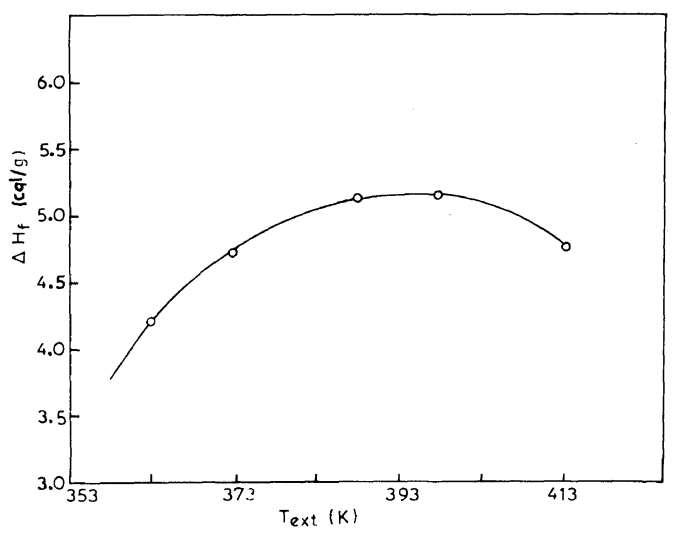

Figure 4. $\Delta H_{\mathrm{f}}$ as a function of $T_{\text {ext }}$ for $R=15$.
$\Delta T_{\mathrm{m}}$ and $\Delta H_{\mathrm{f}}$ plots, it appears natural to suspect a close relationship between these quantities. A plot of $\Delta H_{\mathrm{f}}$ as a function of $\Delta T_{\mathrm{m}}$ is shown in Figure 5. It may be noted that the experimental points, representing measurements on extrudates prepared at different temperatures ( 353 to $398 \mathrm{~K}$ ), lie on a single straight line. The significance of this result is discussed further in the following section.

Kojima et al. ${ }^{5}$ have also obtained similar results but they have attributed the increase in $T_{\mathrm{m}}$ and $H_{\mathrm{f}}$ solely to the increase in crystallinity on extrusion. In the present study, we measured the density of the extrudate for various values of $R$ and found negligible changes (maximum about 0.1 percent). Since the crystallinity is defined here in terms of density, it may therefore be concluded that the extrusion process does not affect this quantity to any appreciable extent. Consequently the changes in $T_{\mathrm{m}}$ and $H_{\mathrm{f}}$ must be due to some other physical process. This point is considered further in the next section.

The dependence of the amorphous orientation function, $f_{\mathrm{am}}$ or $R$ was found to be qualitatively similar to that observed in the case of $\Delta T_{\mathrm{m}}$ and $\Delta H_{\mathrm{f}}$. In Figure 6, the variation of $f_{\text {am }}$ with $R$ is shown for an extrudate obtained at $388 \mathrm{~K}$. On the other hand,

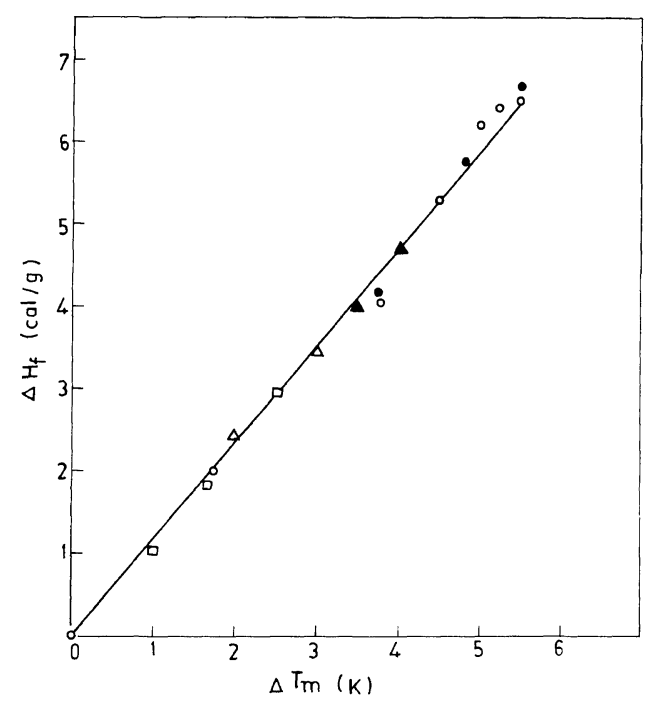

Figure 5. $\Delta H_{\mathrm{f}}$ as a function of $\Delta T_{\mathrm{m}}^{\prime}$. Experimental points, $\boldsymbol{\ominus}, \bigcirc, \Delta, \boldsymbol{\Delta}$, and $\boldsymbol{\square}$, correspond respectively to extrudates prepared at $398,388,373,363$, and $353 \mathrm{~K}$. 


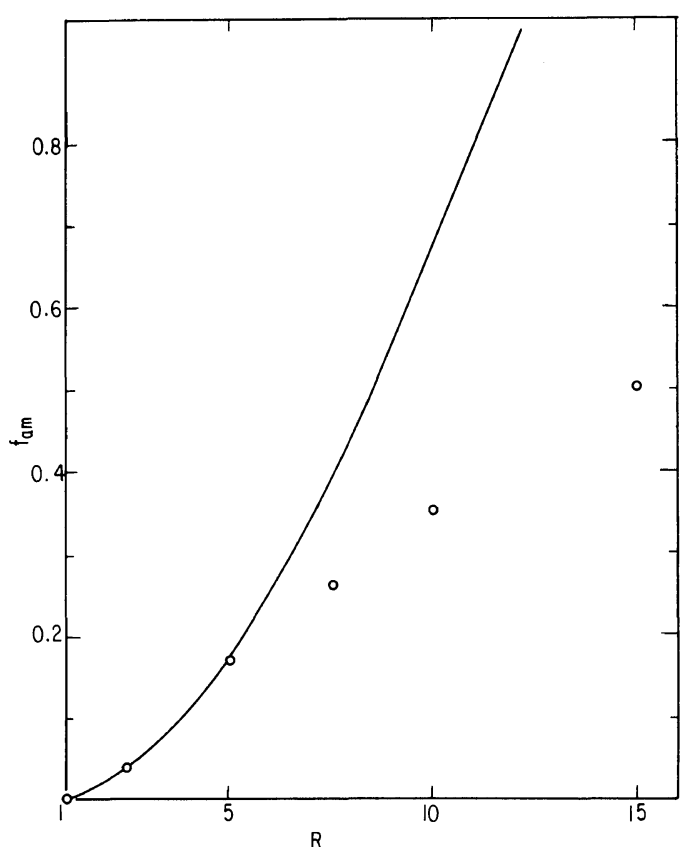

Figure 6. The amorphous orientation function $f_{\mathrm{am}}$ as a function of $R$. The triangles denote the experimental values, the curve represents the variation according to eq 14 of the text. The normalisation of the theoretical and experimental values was carried out for $R=5$.

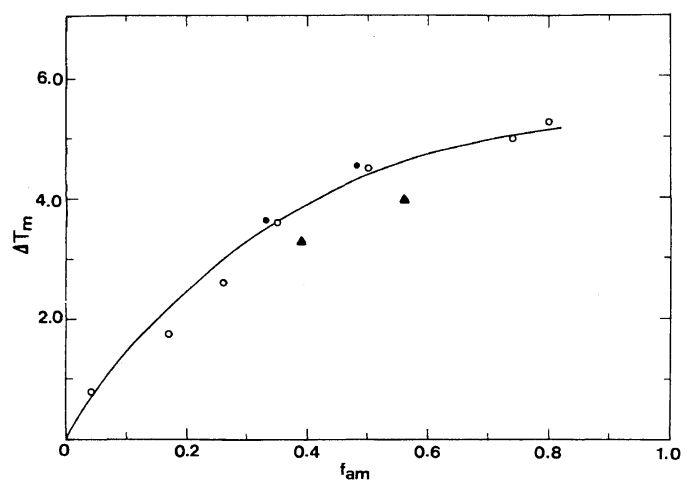

Figure 7. $\Delta T_{\mathrm{m}}$ as a function of $f_{\mathrm{am}}$. The rest is as in Figure 5 .

Figure 7, which shows the relationship between $\Delta T_{\mathrm{m}}$ and $f_{\mathrm{am}}$, serves to underline the relationship between the orientation produced in the amorphous phase and the consequential changes in the melting behavior.

\section{DISCUSSION}

The melting of a semicrystalline polymer occurs when the Gibbs free energies $G_{\mathrm{c}}$ and $G_{\mathrm{a}}$ of its crystalline and amorphous phases, respectively, become equal. Following Sumita et al., ${ }^{4}$ we may write for oriented samples (drawn or extruded),

$$
\begin{aligned}
& G_{\mathrm{c}}=G_{\mathrm{ce}}+\Delta G_{\mathrm{c}} \\
& G_{\mathrm{a}}=G_{\mathrm{ae}}+\Delta G_{\mathrm{a}}
\end{aligned}
$$

where the suffix, e, denotes the equilibrium value while $\Delta G$ gives the change in $G$ brought about by orientation effects. It is easily seen that an increase in $G_{\mathrm{c}}$ lowers $T_{\mathrm{m}}$, while an increase in $G_{\mathrm{a}}$ raises this value. $^{4}$

The changes in crystal form and morphology contribute to $\Delta G_{\mathrm{c}}$ while the orientation of chains in the amorphous phase gives rise to the term $\Delta G_{\mathrm{a}}$. As indicated in the earlier section, the density of polyethylene and hence its degree of crystallinity does not change to any appreciable extent because of extrusion. However, the morphology changes from spherulitic to fibrillar form ${ }^{14}$ and a small fraction of the crystalline phase also undergoes a transformation from the orthorhombic to the monoclinic form. ${ }^{12}$ The contribution of these transformations to $\Delta G_{c}$ in the melting process, however, may be neglected, as has been explained by Sumita et al. ${ }^{4}$ To confirm this, we studied the melting of etched samples. It is expected that after sufficient etching, the chains should be present principally as single traverses of the crystalline regions. ${ }^{15} \mathrm{We}$ found that the melting temperature of such a sample was the same as that of an unoriented sample. Similar results have been reported by Weeks and Porter. ${ }^{16}$ These facts strongly suggest that the changes in $T_{\mathrm{m}}$ and $H_{\mathrm{f}}$ are brought about essentially due to the orientation of chains in the amorphous phase due to extrusion. Sumita et al. have drawn the same conclusion from their investigations carried out with drawn polyethylene. In light of the foregoing conclusions, the observed heat of fusion of an oriented sample may be expressed in the form,

$$
H_{\mathrm{f}}=X H_{\mathrm{fc}}+(1-X) H_{\mathrm{fa}}
$$

where $H_{\mathrm{fc}}$ and $H_{\mathrm{fa}}$ stand respectively for the heats of the transformation of the crystal and the oriented amorphous phase to the normal melt. Sumita et al. ${ }^{4}$ on the basis of certain plausible assumptions, have shown that for oriented samples, 


$$
\Delta H_{\mathrm{fa}}=A \Delta T_{\mathrm{m}}
$$

where $A$ is a constant. Using eq 11 and 12 one finally obtains,

$$
\Delta H_{\mathrm{f}}=A^{\prime} \Delta T_{\mathrm{m}}
$$

where $A^{\prime}=A(1-X)$. This equation agrees with the observed experimental results shown in Figure 5 and thus supports the contention regarding the role of orientation in the amorphous phase. On the otherhand, the results of this paper clearly negate the point of view advanced by Kojima et al. ${ }^{5}$ that the changes in the melting behavior of extruded polyethylene are brought about solely because of the increase in crystallinity. Since, for $R=20$, $\Delta H_{\mathrm{f}}=6.5 \mathrm{cal} \mathrm{g}^{-1}$ (see Figure 2), it follows according to their hypothesis that $X$ undergoes an increase of about 9 percent. An elementary calculation shows that the density of the extrudate consequently should increase by 1.6 percent, but our measurements, as indicated in the last section, showed only an increase of 0.1 percent.

Next, we turn to the consideration of the amorphous orientation function, $f_{\mathrm{am}}$. According to the theory of rubber elasticity, for an $R$ fold linear extension of a polymer network, we have the expression, ${ }^{17}$

$$
f_{\mathrm{am}}=\frac{2 \pi N}{45} \frac{\left(\mu_{0}+2\right)^{2}}{\mu_{0}} \frac{\Delta \alpha}{\Delta n_{\mathrm{a}}^{0}}\left(R^{2}-\frac{1}{R}\right)
$$

where $\mu_{0}$ is the refractive index, and $N$ and $\Delta \alpha$ denote respectively the number of network chains per unit volume and the polarizability difference. The curve in Figure 6 represents the variation of $f_{\mathrm{am}}$ with $R$, according to eq 14 where the normalisation of the theoretical and experimental values has been carried out for $R=5$. It is observed that the theory represents the experimental values quite well for low $R$ values. Departures for $R>5$ may be expected and can be attributed to the break down of the theory of rubber elasticity for large deformations.

Finally, we may refer to the significance of the plot of $T_{\mathrm{m}}$ versus $f_{\mathrm{am}}$, shown in Figure 7. The data points representing the results of measurements with extrudates prepared at different temperatures, lie on a single curve just as in the case of $\Delta T_{\mathrm{m}}$ versus $\Delta H_{\mathrm{f}}$ plot of Figure 5. It may thus be concluded that, for a given polymer $\Delta T_{\mathrm{m}}$ and $\Delta H_{\mathrm{f}}$ depend only on the value of $f_{\mathrm{am}}$. This further confirms the earlier observation that changes in the melting character of high-density polyethylene are governed essentially by the extent of orientation in the amorphous phase.

Acknowledgements. The authors acknowledge with thanks the U.N.D.P. assistance in the form of certain items of equipment. This work was supported by a research grant received from the University Grants Commission (India).

\section{REFERENCES}

1. P. J. Flory, L. Mandelkern, and H. K. Hall, J. Am. Chem. Soc., 73, 2532 (1951).

2. R. Chiang and P. J. Flory, J. Am. Chem. Soc., 83, 2857 (1961).

3. K. Sakurai, T. Oota, K. Miyasaka, and K. Ishikawa, J. Polym. Sci., Polym. Phys. Ed., 14, 1527 (1976).

4. M. Sumita, K. Miyasaka, and K. Ishikawa, J. Polym. Sci., Polym. Phys. Ed., 15, 837 (1977).

5. S. Kojima, C. R. Desper, and R. S. Porter, J. Polym. Sci., Polym. Phys. Ed., 16, 1721 (1978).

6. Y. R. Midha and V. S. Nanda, Macromolecules, 10, 1031 (1977).

7. R. K. Jain, R. N. Gupta, and V. S. Nanda, J. Macromol. Sci., Phys., B11, 411 (1975).

8. K. Dhawan, P. C. Jain, and V. S. Nanda, to be published.

9. R. S. Stein, J. Polym. Sci., 31, 327 (1958).

10. S. Hoshino, J. Powers, D. G. LeGrand, H. Kawai, and R. S. Stein, J. Polym. Sci., 58, 185 (1962).

11. C. W. Bunn and R. Daubney, Trans. Faraday Soc., 50, 1173 (1954).

12. K. Nakayama and H. Kanetsuna, J. Mater. Sci., 10, 1105 (1975).

13. F. A. Bettelhem and R. S. Stein, J. Polym. Sci., 27, 567 (1958).

14. G. Meinel and A. Peterlin, J. Polym. Sci., A-2, 9, 67 (1971).

15. A. Keller, J. Polym. Sci., A-2, 9, 1793 (1971).

16. N. E. Weeks and R. S. Porter, J. Polym. Sci., Polym. Phys. Ed., 13, 2049 (1975).

17. A. Ciferri, C. A. J. Hoeve, and P. J. Flory, J. Am. Chem. Soc., 83, 1015 (1961). 